\title{
Preventive effects of statin therapy in coronary artery diseases: Current controversy
}

\author{
Yasser Ali Kamal ${ }^{1}$, Seham Abdel-Wakeel Abdel-Gaber ${ }^{2}$
}

Kamal YA, Abdel-Gaber SAW. Preventive effects of statin therapy in coronary artery diseases: Current controversy. Curr Res Integr Med 2018;3(S1): 5-10.

The role of statins for primary and secondary prevention of coronary artery disease (CAD), is extensively investigated. This review of literature highlights the current controversies of the research regarding this role. Despite the great impact of statins on outcomes during management of CAD, many issues need further investigation. A debate remains about the biomarkers of cardiovascular pleiotropic effects, stratification of high-risk persons eligible for therapy, evaluating cost-effectiveness, patient factors enhancing benefit-based therapy, long-term benefits, combination therapy, and residual risk after coronary revascularization. Coronary artery calcium (CAC) scoring is a useful tool, when combined to clinical cardiovascular risk scores, in order to guide the shared decision for prescription of statins in asymptomatic persons. A balance approach remain to reserve high-doses of statins for patients with ACS and when the therapeutic target of LDL-C at $70 \mathrm{mg} / \mathrm{dl}$ could not obtained with moderate-doses. Future powered studies are required to detect biomarkers of statin therapy, eligibility for the prophylactic use, reasons for real-life non-adherence with statins, predictors of the statin effects in combination with other lipid-lowering agents, factors enhance the benefit-based therapy, ideal therapeutic doses, and the reasons of the residual risk. and respiratory systems, cardiac anomalies should be considered. Our case of congenital pulmonary vein stenosis presented with refractory wheezing from pulmonary venous obstruction. Unfortunately, this disorder has an unrelenting course. Even with early diagnosis and current surgical management, the outcome is poor.

Key Words: Coronary artery disease; statin;, prevention; acute coronary syndrome; coronary revascularization

Abbreviations: ACC/AHA: American College of Cardiology/American Heart Association; ACS: Acute Coronary Syndrome; AF: Atrial Fibrillation; Akt: Protein Kinase B; CABG: Coronary Artery Bypass Grafting; CAC: Coronary Artery Calcium; CAD: Coronary Artery Disease; DES: Drug-Eluting Stent; DM: Diabetes Mellitus; eNOS: Endothelial-Type Nitric Oxide Synthase; EPA: Eicosapentaenoic Acid; EPC: Endothelial Progenitor Cells; ESC: European Society of Cardiology; HDL-C: High Density Lipoprotein Cholesterol; HMG-CoA: 3-Hydroxy-3-Methyl-Glutaryl-Coenzyme A; hsCRP: High Sensitivity C-Reactive Protein; IL: Interleukin; LDL-C: Low-Density Lipoprotein Cholesterol; Lp (a): Lipoprotein (a); MACE: Major Adverse Cardiovascular Event; MESA: Multi-Ethnic Study of Atherosclerosis; MI: Myocardial Infarction; mTOR: Mammalian Target of Rapamycin; NC: Necrotic Core; NSTE-ACS: Non-ST Elevation Acute Coronary Syndrome; PCI: Percutaneous Coronary Intervention; PI3K: Phosphoinositide 3-Kinase; QALY: Quality-Adjusted Life Year
Statins inhibit hepatic biosynthesis of cholesterol where they prevent mevalonate formation, and inhibit 3-hydroxy-3-methyl-glutaryl-coenzyme A (HMG-CoA) (1-2). Through the last decades, research targets the protective cardiovascular effects of statins (3-4). Statins have desired roles preventing primary and secondary all-cause death and major cardiovascular events of coronary artery disease (CAD) (5). Protecting against atherosclerotic cardiovascular disease, statin therapy is effective with modification of risk factors (6).

The favorable pleiotropic effects occur through increasing expression of atheroprotection genes, inhibiting inflammatory markers, protecting endothelium, enhancing plaques stability, inhibiting platelets aggregation, increasing nitric oxide bioavailability, decreasing circulating oxidative stress and inflammatory biomarkers, and inhibiting thrombogenesis $(2,7)$.

Variable recommendations of statins in high-risk CAD patients exist. Many guidelines recommend low-density lipoprotein-cholesterol (LDL-C) goal at $70 \mathrm{mg} / \mathrm{dl}(8-9)$, but the guideline of American College of Cardiology/ American Heart Association (ACC/AHA) supports high-intensity statins and repeated lipid measures (10).

A debate exists about prescribing patterns, eligible patients, high-intensity doses (11-13), combined lipid-lowering therapy, improved outcomes following coronary surgery or intervention (14-15). Therefore, our review of the contemporary literature concerns contemporary status and debates of statin therapy in CAD patients. Our methods of constituting this narrative review include searching MEDLINE with a limit of publication dates between January and December 2017, using search terms of (statins) and (coronary artery disease) which yield 319 trials, of which 104 studies were relevant to the aim of our review.

\section{Impact of Statins on Biomarkers}

The current research proceeds to investigate the role of statins in repairing, stabilization, and regression of coronary artery lesions, which form a cornerstone in inhibition of cardiac events (16). However, the mechanisms rather than lowering LDL-C to produce beneficial cardiovascular pleiotropic effects, remain multifactorial and unclear.

Statins can modify the number and function of endothelial progenitor cells (EPC) which has a critical role in vascular repairing. The currently main proposed mechanisms include: (1) reduction of micro non-coding RNAs in EPC, helping its differentiation and mobilization; (2) enhancing endothelialtype nitric oxide synthase (eNOS) associated with EPC mobilization; and (3) stimulating the phosphoinositide 3-kinase (PI3K)/protein kinase B (Akt)/ mammalian target of rapamycin (mTOR) pathway to activate eNOS, and hence enhancing mobilization and number of EPC (17).

Statins exhibit potent anti-inflammatory effects on atherogenesis, resulting in reduction of the circulating levels of high sensitivity C-reactive protein (hsCRP) and adhesion molecules (18). A possible mechanism underlying plaque stabilization is reduction of hsCRP with decreased necrotic core (NC), and hence reinforced fibrous cap, 12-month after rosuvastatin therapy (19). Interesting findings demonstrated a beneficial effect of statins on depression, one year following ACS due to attenuated pro-inflammatory cytokines; interleukin (IL)-6 and IL-18 (20).

A debate exists concerning Lipoprotein (a) [Lp (a)] as a biomarker for longterm statin effects on atheroma volume. A recent study indicates modifying long-term statin therapeutic effects on $\mathrm{Lp}$ (a) by traditional risk factors including primary hypertension, diabetes mellitus (DM), LDL-C and high density lipoprotein cholesterol (HDL-C) (21). Also, the recent insights from [Study of coronary atheroma by intravascular ultrasound: Effect of Rosuvastatin Versus Atorvastatin] (SATURN) revealed no association of Lp (a) levels with progression of coronary atheroma in patients with CAD using intensive statins and have low on-treatment LDL-C levels (22).

The increased vitamin D levels and reduced platelet reactivity are potential indicators of the pleiotropic high-intensity statins benefit during dual

${ }^{1}$ Minia Cardiothoracic University Hospital, Egypt, 2Faculty of Medicine, Minia University, Egypt

Correspondence: Kamal YA, Minia Cardiothoracic University Hospital, El-Minya El-Jadeda, Postal Code: 61768, Minia, Egypt,, Telephone 20-01123234736, e-mail yaser_ali_kamal@yahoo.com

Received: March 15, 2018, Accepted: April 17, 2018, Published: April 27, 2018

OPEN $\bigcirc$ Access

This open-access article is distributed under the terms of the Creative Commons Attribution Non-Commercial License (CC BY-NC) (http:// creativecommons.org/licenses/by-nc/4.0/), which permits reuse, distribution and reproduction of the article, provided that the original work is properly cited and the reuse is restricted to noncommercial purposes. For commercial reuse, contact reprints@pulsus.com 
antiplatelet therapy receiving high-intensity statins following ACS or elective percutaneous coronary intervention (PCI) (23). Another emerging biomarker of CAD, namely omentin-1, has a dose-dependent increase with atorvastatin therapy. A dose of $40 \mathrm{mg}$ has a greater effect on levels of omentin-1 than does atorvastatin at $20 \mathrm{mg}(24)$.

\section{Prescription Strategies and Eligibility for Therapy}

Primary Prevention of Cardiovascular Disease: Low and moderate-dose statins have a confirmed benefit for individuals aged $40-75$ years old with a cardiovascular risk factor and 10 -year event risk of $7.5-10 \%$ (25). The ACC/AHA guideline-based class-I recommendations include persons have 10-year risk of CVD $\geq 7.5 \%$, DM, and LDL-C $\geq 190 \mathrm{mg} / \mathrm{dL}$ (26). A recent Multi-Ethnic Study of Atherosclerosis (MESA) considers these indications as a superior screening tool for subclinical and clinical CVD (27). The costeffectiveness analysis revealed that 10-years universal statin use, starting at 2016, vary moderately considering different risk indications and statin toxicity, and affected greatly by pill burden (28).

An interesting point of the current research is to determine possible markers that may help identify individuals eligible for statin therapy. Coronary artery calcium (CAC) score $>100$ has a useful role in decision making for statin therapy. It can help identify individuals at high risk for cardiovascular disease (CVD) and target guideline-based statin therapy to persons likely to have the most benefit (29). Moreover, CAC score $>100$ associated with mortality whether statins recommended, considered, or non-recommended (30). In addition, when high-intensity statins required in asymptomatic individuals, elevated CAC score $\geq 300$ associated with age, male gender, and fasting blood glucose, thus, individualization of therapy and lifestyle modification will be useful in this instance (31).

Recent studies reported CAC score $<100$ common in persons taking statins in accordance to AHA/ACC and European Society of Cardiology (ESC) guidelines. This may change decision-making of statin therapy, by matching clinical risk to atherosclerotic plaque burden (32), with reclassification of the risk in $50 \%$ of people with estimated 10 -year CVD risk of $5 \%$ to $20 \%$ (33). Thereafter, measuring CAC score gains its significance to find the real risk and then avoiding subjects with low 10-year risk for CVD (32-33). This may support individual-physician discussion when decide long-term statin therapy.

A cost-effectiveness study of using CAC score to guide long-term statin therapy compared with treating all patients eligible for statins according to 2013 ACC/AHA guidelines, suggesting that the economic value of both approaches are similar. Therefore, clinicians should account for each preference in context of shared decision-making when choosing good strategy to guide statin decisions (34).

Secondary Prevention of Cardiovascular Events: Statins are still recommended as the first-line for secondary prevention after ACS, as the early intensive therapy with atorvastatin $80 \mathrm{mg}$ daily decreases the burden of atherosclerotic plaques, with reduced time to first occurrence of death, non-fatal myocardial infarction (MI), resuscitated cardiac arrest, or hospitalization for unstable angina, hard cardiovascular events, and the need for revascularization in symptomatic patients (35-37). Moreover, statins can decrease morbidity during the acute phase of ACS in patients on chronic pre-treatment, as they can decrease plaque rupture particularly in those with non-ST segment elevation ACS (38). On the other hand, patients with ACS on no-statin therapy have an increased risk of CVD-related death overtime, which remains constant for those on high-intensity statins (39).

Statin effects after ACS correlated with accumulated dose and therapy duration (40), and it is proportional to the achieved LDL-C (41). The influences on lipid levels and cholinesterase activity further confirm the beneficial effects of intensive statin therapy in patients with CAD (42). Moreover, the highest overall quality-adjusted life year (QALY) gain against acceptable costs confirmed the cost-effectiveness of intensive lipid-lowering therapy with statins (43).

Despite the beneficial effects of statins, a dichotomy exists between intention to prescribe and real prescribing of therapy (44). Less than $40 \%$ of CAD statin-treated patients attained their recommended LDL-C and non-HDL-C goals due to irregular use and unscheduled dosage (45), with discontinuation rates of 53.7 and $84.3 \%$ at one and three-year follow-up, respectively, after ACS (46).

Suboptimal statin use is common early after non-ST elevation acute coronary syndrome (NSTE-ACS) due to muscular symptoms, therefore, keep, and enhance use of high-potency statin therapy can improve outcomes (47). Good adherence to statin therapy through 6 months after ACS without declined dosage is important to prevent major adverse cardiovascular events (MACEs), with recommendations to give higher tolerable dosage (48).

Patient factors associated with non-use of high-potency statins after ACS include age older than 75 years, female gender, renal dysfunction, heart failure during hospital admission, and lower LDL-C (49). In addition, statin intolerance is an important factor that affect adherence to statins after hospitalization for MI and associated with an increased risk for recurrent MI and CAD events over the year following hospital discharge, in comparison to those on high-intensity statins (50).

\section{Effects of Combined Therapy}

Combination with Ezetimibe: Ezetimibe is a non-statin lipid-altering drug reduces intestinal cholesterol absorption. Combination of statins with ezetimibe could help attainment of guideline LDL-C and non-HDL-C goals (45). The greater effects of combination therapy explained partly by its more significant anti-inflammatory effect than mono-therapy (51). Other investigated mechanisms underlying the effects of combination therapy are ameliorating endothelial dysfunction in stented coronary arteries, and larger decreases in oxysterol levels (52). However, the anti-atherosclerotic mechanisms of ezetimibe and ezetimibe-statin combination therapy remains controversial in a recent randomized trial, as it did not result in a significant change in coronary plaque regression compared with statin alone (53).

In a recent 6-year population-based cohort study, using both agents together for patients with ACS and multiple comorbidities associated with lower risks of re-hospitalization for ACS and revascularization (54). Moreover, adding ezetimibe to simvastatin reduces the risk of ischemic stroke after ACS, particularly in patients with a prior stroke (55).

In comparison to intensive statin therapy in patients with ACS, statin ezetimibe combination therapy has a greater effect on lowering LDL-C, drugrelated adverse events, percentage of non-high density lipoprotein-cholesterol (non-HDL-C), and cholesterol absorption (56-58).

Examining effects of statin-ezetimibe combination in special patient groups revealed similar response for both men and women (59), less liver dysfunction than double-dose statin in very elderly patients with ACS (60), and better role than mono-therapy associated with reducing blood lipid levels and improving plaque stability in diabetic patients with CAD (61).

Combination with Eicosapentaenoic Acid: Eicosapentaenoic acid (EPA), an omega-3 fatty acid, lowers plasma triglycerides, reduces levels of proinflammatory cytokines and chemokines, and may decrease coronary plaque vulnerability and prevent plaque progression (62). In comparison to statin monotherapy, combination of statins with EPA therapy during the first 24 hours after PCI reduces inflammation and ventricular arrhythmia, and reduces cardiovascular events after primary PCI if early initiated in patients with ACS (63).

Combination therapy reinforces plaque stabilization particularly in patients with stable angina, and appears a promising option in patients under intensive and strong statin therapy to decrease residual CAD risk (64-65), particularly in patients with a higher baseline risk for residual disease (66).

Combination with Nutraceuticals: Nutraceuticals are combination of natural components. Similar to ezetimibe, nutraceuticals are alternative therapy to statins in patients intolerant to high-dose statins, as they lower LDL-C concentration when used alone or in association with other agents (67).

Low-dose statin combination with nutraceuticals such as Armolipid Plus (red yeast rice, policosanol, berberine, folic acid, coenzyme Q10 and astaxanthin) represents a valuable therapeutic option in patients with CAD and high-dose statin intolerance (68).

\section{Impact on Outcomes after Coronary Revascularization}

Impact on Outcome after Percutaneous Intervention: In the current actual practice, long-term statin therapy before primary PCI improves epicardial perfusion in patients with stable angina (69) and treatment outcomes of acute MI (70). Both rosuvastatin and atorvastatin are suitable and well tolerated to control lipid levels and prevent risk of cardiovascular events after PCI (71). The benefit of high-dose atorvastatin to decrease the risk of MI and cardiovascular adverse events supports its current use as an adjunct to aid PCI (72).

The loading high-dose of rosuvastatin before PCI in patients with ACS has more beneficial effect than the conventional dose, however, the limited quality of studies indicates further evidence (73). In addition, a high-dose of 
rosuvastatin can delay ventricular remodeling, inhibit malignant remodeling, improve left ventricular systolic function, and decrease the prevalence of adverse events, 3 months after emergent PCI (74).

A controversy exists about the effect of gender on long-term outcomes of statin therapy after PCI. In a recent study with a median follow-up period of 6.3 years, the cumulative rate of MACE were higher in women than in men, however, there was no significant association of female gender with MACE after adjusting for age and other risk variables (75).

The primary targets for maximizing the beneficial effects of statin therapy after PCI are elderly patients and those with chronic kidney disease (76). Even in patients older than 80 years, statin therapy through 5 years after PCI associated with significant reduced risk for cardiovascular events (77). In patients with ACS, received either $40 \mathrm{mg}$ simvastatin or $20 \mathrm{mg}$ atorvastatin daily, for at least 6 months after PCI, the rate of declined renal function was similar in both drug groups $(<27 \%)(78)$, however, comparative renoprotective effects of statins need further evaluation.

In patients with diabetes mellitus, which affects the prognosis of PCI, a high intensity statin therapy increases EPC levels, decreases in-stent neointima area and volume, and does not affect the degree of stent re-endothelialization at 3 months after drug-eluting stent (DES) implantation (79). Interestingly, pitavastatin therapy (4 $\mathrm{mg}$ daily) has more favorable effects than atorvastatin (20 mg daily) on glucose control in patients with non-ST elevation ACS received successful PCI (80).

In summary, literature shows favorable outcomes after PCI whether statins prescribed as long-term pretreatment or as an adjunct to PCI. However, a controversy exists on the use of high-dose rosuvastatin and the proper type of loading dose before PCI. Despite the beneficial effects of statins after PCI in elderlies, chronic kidney disease and diabetics, there is a need for further studies to compare the effects of different statin types and doses.

Impact on Outcome after Coronary Artery Bypass Grafting: Preoperative statin therapy has a beneficial effect to decrease risk of mortality after coronary artery bypass grafting (CABG). However, little data exists on optimal dose and timing of statin therapy. A recent study demonstrated that both preoperative statin use $<24$ hours and a dose of $>20 \mathrm{mg}$ associated with decreased 30-day all-cause mortality after CABG (81). In contrary, another recent study reported dose-independent pattern of statin therapy to decrease in-hospital mortality and MACE in patients undergoing CABG for ACS (82).

The biggest beneficiary of statin pretreatment in CABG is reduction of postoperative atrial fibrillation (AF), attributed to modulation of systemic inflammatory markers (cytokines, C-reactive protein) (83). A controversy remains about the effect of preoperative statin therapy on renal outcomes after isolated CABG. In a recent study, although preoperative statin therapy associated with lower risk of postoperative renal dysfunction and the need for renal replacement, it did not decrease the risk of acute kidney injury (84). Interestingly, pretreatment with atorvastatin reduced the risk of bleeding and blood products use after on-pump CABG, probably due to a reduction in the postoperative inflammatory response. A recommendation remains for statin continuation at the highest tolerable dose before cardiac surgery (85), although further well-designed studies required.

\section{Predicting Residual Risk for CAD}

The vascular response to statin therapy in patients with CAD, due to stabilization of thin-cap fibroatheroma, associated with favorable cardiovascular outcomes. Residual risk for CAD remains due to lack of the favorable vascular response. Chronic kidney disease is an independent clinical predictor for unfavorable vascular response to statin therapy (86).

Recent investigated biomarkers associated with residual risk of CAD following coronary revascularization despite statin treatment determined that increased cholesterol absorption marker and apoB48 concentration lead to de-novo lesions (87). In addition, higher levels of $\mathrm{Lp}$ (a) carries a residual cardiovascular risk for adverse events among $\mathrm{CAD}$ patients receiving intensive statin therapy after PCI (88), however, correlation of measured $\mathrm{Lp}$ (a) levels and coronary atheroma progression was absent in CAD patients prescribed long-term maximally intensive statin therapy who have low ontreatment LDL-C levels (22).

\section{SUMMARY}

A controversy exists regarding biomarkers of the pleiotropic effects associated with statin therapy in CAD patients. The investigated mechanisms include vascular repairing by enhanced endothelial progenitor cells, plaque stabilization due to reduction of hs-CRP and necrotic core, and attenuated cytokines effect on depression following ACS. Emerging biomarkers of statin effects include lipoprotein (a), vitamin D levels, and serum omentin-1 levels.

The targeted universal statin use for primary prevention of CVD may depend on the inutility caused by pill burden. Quantification of coronary artery calcium score in addition to the guidelines improves stratification of subjects at high risk for cardiovascular events. The CAC score is an important complementary tool for risk stratification in asymptomatic persons need high-intensity statins. The role of each preference for approach of therapy in context of shared decision-making with clinicians supported by the similar economic values of CAC scoring and guideline-based primary prevention for all eligible persons.

Statins are the first-line treatment for the secondary prevention after ACS. In patients on chronic pre-treatment, statins have beneficial effects on morbidity during the acute phase of ACS. The beneficial effect of statins for secondary prevention in CAD patients correlated with its dose and therapy duration. The cost-effectiveness results with the highest overall quality-adjusted life year (QALY) gain against acceptable costs adds an economic benefit of intensive statin therapy. A contrast exists between intention to prescribe and real prescribing behaviors of high-intensity dose statin for patients with CAD that may affect guideline recommended cholesterol goals. Discontinuation of statins or non-adherence carries an adverse prognosis after discharge of patients with ACS. Non-use of high-potency statins after ACS associated with elder age, female sex, renal dysfunction, heart failure during admission, lower LDL-C, and statin intolerance.

The combination of statins with other agents such as ezetimibe, eicosapentaenoic acid, and nutraceuticals is a promising option to increase benefits of lipid lowering agents or subsiding high-dose statins. However, the mechanisms of subsequent effects due to combination with ezetimibe remain unclear. Adding ezetimibe to statins is beneficial for patients with ACS and multiple comorbidities, and may replace intensive statin therapy. Very elderly and diabetic patients are good targets for combination therapy. Early initiation of statins with eicosapentaenoic acid after ACS has a significant role to decrease intensive statin therapy and residual CAD risk. Nutraceuticals is a valuable therapeutic option allowing the use of low-dose statins instead of intolerable high-doses.

The beneficial effects of preoperative statin therapy on outcomes after CABG are widely reported. A controversy remains about optimal dose and timing of therapy, in addition to the effect of statins on renal outcomes. Modulation of systemic inflammatory markers may explain the beneficial effect of statin pretreatment on occurrence of AF after CABG. An emerging benefit of statin therapy is reduction of bleeding risk after on-pump CABG but it requires more evidence.

The residual risk remains for CAD despite statin therapy. Debate remains on proper clinical factors and biomarkers associated with the residual risk for CAD undergoing coronary revascularization despite statin therapy. The current investigated factors underlying residual-risk include chronic kidney disease, increased cholesterol absorption marker, apoB48 concentration, and higher serum levels of lipoprotein (a).

\section{CONCLUSION}

Statins have a great impact on primary and secondary prevention of CAD, however, many issues need an investigation. The points of debate and inconclusive data may be related to variation in study design, presence of scant randomized trials comparing the effects of statin therapy, variation in dose and type of statins, and absence of universal biomarkers of statin cardiovascular effects.

As proved in the contemporary literature that CAC scoring can reclassify asymptomatic individuals at risk for ASCVD before initiating statin therapy, we recommend more application of CAC scoring for shared decision making in adherence to clinical scoring systems, particularly in moderate risk persons (5-20\% 10-year ASCVD risk) and in those with a family history of premature CAD. As a controversy exists about the adverse events of high-dose statins, a recommendation for balance therapy remains to reserve these doses for patients with ACS and when the therapeutic target of LDL-C at $70 \mathrm{mg} / \mathrm{dl}$ could not obtained with moderate-doses.

Moreover, we recommend future studies to detect the emerging biomarkers of statin pleiotropic effects, persons eligible for therapy, reasons for the gap between recommendations of guidelines and real-life prescription of statins, predictors of the statin effects in combination with ezetimibe, pre-procedural factors enhancing benefit-based therapy, ideal doses of statins before revascularization, and reasons underlying the residual risk after therapy. 


\section{FUNDING SOURCE}

The authors received no funding for this work.

\section{CONFLICT OF INTEREST}

The authors declare that they have no competing interests.

\section{REFERENCES}

1. Istvan ES, Deisenhofer J. Structural mechanism for statin inhibition of HMG-CoA reductase. Science. 2001;292:1160-4.

2. Habon T, Toth K. Pre-treatment with statins for coronary intervention: Pleiotropy of statins or effect of LDL-cholesterol reduction? Korean Circ J. 2016;46: 468-71.

3. Pedersen TR. Pleiotropic effects of statins: Evidence against benefits beyond LDL-cholesterol lowering. Am J Cardiovasc Drugs. 2010;10:10-17.

4. Oesterle A, Laufs U, Liao JK. Pleiotropic effects of statins on the cardiovascular system. Circ Res. 2017;120:229-43.

5. Naci H, Brugts JJ, Fleurence R, et al. Comparative benefits of statins in the primary and secondary prevention of major coronary events and all-cause mortality: a network meta-analysis of placebo-controlled and active-comparator trials. Eur J Prev Cardiol. 2013;20:641-57.

6. Taylor F, Huffman MD, Macedo AF, et al. Statins for the primary prevention of cardiovascular disease. Cochrane Database Syst Rev. 2013;1:CD004816.

7. Zhou Q, Liao JK. Statins and cardiovascular diseases: From cholesterol lowering to pleiotropy. Curr Pharm Des. 2009;15(5):467-78.

8. Grundy SM, Cleeman JI, Merz CN, et al. Implications of recent clinical trials for the National Cholesterol Education Program Adult Treatment Panel III guidelines. Circulation. 2004;110:227-39.

9. Reiner Z, Catapano AL, De Backer G, et al. ESC/EAS guidelines for the management of dyslipidaemias: The task force for the management of dyslipidaemias of the European Society of Cardiology (ESC) and the European Atherosclerosis Society (EAS). Eur Heart J. 2011;32(14):1769-818.

10. Stone NJ, Robinson JG, Lichtenstein AH, et al. 2013 ACC/AHA guideline on the treatment of blood cholesterol to reduce atherosclerotic cardiovascular risk in adults: A report of the American College of Cardiology/American Heart Association Task Force on Practice Guidelines. J Am Coll Cardiol. 2014;63(25 Pt B):2889-934.

11. Pursnani A, Massaro JM, D’Agostino RB Sr, et al. Guideline-based statin eligibility, coronary artery calcification, and cardiovascular events. JAMA. 2015;314(2):134-41.

12. Valentino M, Al Danaf J, Panakos A, et al. Impact of the 2013 American College of Cardiology/American Heart Association cholesterol guidelines on the prescription of high-intensity statins in patients hospitalized for acute coronary syndrome or stroke. Am Heart J. 2016;181:130-6.

13. Watanabe E, Yamaguchi J, Arashi H, et al. Effects of statin versus the combination of ezetimibe plus statin on serum lipid absorption markers in patients with acute coronary syndrome. J Lipids. 2015;2015:109-158.

14. Peng JA, Ancock BP, Conell C, et al. Nonutilization of statins in a community-based population with a history of coronary revascularization. Clin Ther. 2016;38(2):288-96.e2.

15. Matsue Y, Yoshida K, Nagahori W, et al. Peripheral microvascular dysfunction predicts residual risk in coronary artery disease patients on statin therapy. Atherosclerosis. 2014;232(1):186-90.

16. Miyauchi K, Daida H. Clinical significance of intensive lipid-lowering therapy using statins in patients with coronary artery disease: LDLcholesterol: The lower, the better; is it true for Asians? (Pro). Circ J. 2010;74(8):1718-30.

17. Sandhu K, Mamas M, Butler R. Endothelial progenitor cells: Exploring the pleiotropic effects of statins. World J Cardiol. 2017;9(1):1-13.

18. Diamantis E, Kyriakos G, Quiles-Sanchez LV, et al. The anti-inflammatory effects of statins on coronary artery disease: An updated review of the literature. Curr Cardiol Rev. 2017;13(3):209-16.
19. Kwon O, Kang SJ, Kang SH, et al. Relationship between serum inflammatory marker levels and the dynamic changes in coronary plaque characteristics after statin therapy. Circ Cardiovasc Imaging. 2017;10(7): e005934.

20. Kim SW, Kang HJ, Bae KY, et al. Interactions between pro-inflammatory cytokines and statins on depression in patients with acute coronary syndrome. Prog Neuropsychopharmacol Biol Psychiatry. 2018;80(Pt C):250-4.

21. Xu MX, Liu C, He YM, et al. Long-term statin therapy could be efficacious in reducing the lipoprotein (a) levels in patients with coronary artery disease modified by some traditional risk factors. J Thorac Dis. 2017;9(5):1322-32.

22. Puri R, Ballantyne CM, Hoogeveen RC, et al. Lipoprotein(a) and coronary atheroma progression rates during long-term high-intensity statin therapy: Insights from SATURN. Atherosclerosis. 2017;263:137-44.

23. Verdoia M, Pergolini P, Rolla R, et al. Impact of high-dose statins on vitamin $\mathrm{D}$ levels and platelet function in patients with coronary artery disease. Thromb Res. 2017;150:90-5

24. Chen Q, Shang X, Yuan M, Liang L, Zhong X. Effect of atorvastatin on serum omentin-1 in patients with coronary artery disease. Coron Artery Dis. 2017;28(1):44-51.

25. Draft Recommendation Statement: Statin use for the primary prevention of cardiovascular disease in adults: Preventive medication. U.S. Preventive Services Task Force. Accessed on 20 December 2017.

26. Stone NJ, Robinson JG, Lichtenstein AH, et al. American College of Cardiology/American Heart Association Task Force on Practice Guidelines. 2013 ACC/AHA guideline on the treatment of blood cholesterol to reduce atherosclerotic cardiovascular risk in adults: A report of the American College of Cardiology/American Heart Association Task Force on Practice Guidelines. Circulation. 2014;129(25 suppl 2):S1-S45.

27. Flueckiger P, Qureshi W, Michos ED, et al. Guideline-based statin/ lipid-lowering therapy eligibility for primary prevention and accuracy of coronary artery calcium and clinical cardiovascular events: The multiethnic study of atherosclerosis (MESA). Clin Cardiol. 2017;40(3):163-9.

28. Heller DJ, Coxson PG, Penko J, et al. Evaluating the impact and cost effectiveness of statin use guidelines for primary prevention of coronary heart disease and stroke. Circulation. 2017;136(12):1087-98.

29. Mortensen MB, Falk E, Li D, et al. Statin trials, cardiovascular events, and coronary artery calcification: Implications for a trial-based approach to statin therapy in MESA. JACC Cardiovasc Imaging. 2017;S1936$878 \mathrm{X}(17): 30405-9$

30. Han D, Hartaigh B, Lee JH, et al. Assessment of Coronary artery calcium scoring for statin treatment strategy according to ACC/AHA guidelines in asymptomatic Korean adults. Yonsei Med J. 2017;58(1):82-9.

31. Lee DH, Youn HJ, Jung HO, et al. Coronary artery calcium score plays an important role for cardiovascular risk stratification in the statin benefit groups of asymptomatic individuals. Lipids Health Dis. 2017;16(1):172.

32. Mahabadi AA, Möhlenkamp S, Lehmann N, et al. CAC score improves coronary and CV risk assessment above statin indication by ESC and AHA/ACC primary prevention guidelines. JACC Cardiovasc Imaging. 2017;10(2):143-53

33. Michos ED, Blaha MJ, Blumenthal RS. Use of the coronary artery calcium score in discussion of initiation of statin therapy in primary prevention. Mayo Clin Proc. 2017;92(12):1831-41.

34. Hong JC, Blankstein R, Shaw LJ, et al. Implications of coronary artery calcium testing for treatment decisions among statin candidates according to the ACC/AHA cholesterol management guidelines: A costeffectiveness analysis. JACC Cardiovasc Imaging. 2017;10(8):938-52.

35. Vavuranakis M, Kariori M, Siasos G, et al. Statins in acute coronary syndromes. Curr Pharm Des. 2017.

36. Schwartz GG, Olsson AG, Ezekowitz MD. Effects of atorvastatin on early recurrent ischemic events in acute coronary syndromes: The MIRACL study: A randomized controlled trial. JAMA 2001;285:1711-18.

37. Schwartz GG, Fayyad R, Szarek M, et al. Early, intensive statin treatment reduces 'hard' cardiovascular outcomes after acute coronary syndrome. Eur J Prev Cardiol. 2017;24(12):1294-6. 
38. Gili S, Iannaccone M, Colombo F, et al. Effects of statins on plaque rupture assessed by optical coherence tomography in patients presenting with acute coronary syndromes: Insights from the optical coherence tomography (OCT)-FORMIDABLE registry. Eur Heart J Cardiovasc Imaging. 2017.

39. Rockberg J, Jørgensen L, Taylor B, et al. Risk of mortality and recurrent cardiovascular events in patients with acute coronary syndromes on high intensity statin treatment. Prev Med Rep. 2017;6:203-9.

40. Huang CY, Lin TT, Yang YH, et al. Effect of statin therapy on the prevention of new-onset acute coronary syndrome in patients with rheumatoid arthritis. Int J Cardiol. 2017;S0167-5273(17):33898-6.

41. Miyauchi K, Daida H. Clinical significance of intensive lipid-lowering therapy using statins in patients with coronary artery disease: LDLcholesterol: the lower, the better; is it true for Asians? (Pro). Circ J. 2010;74(8):1718-30.

42. Pytel E, Bukowska B, Koter-Michalak M, et al. Effect of intensive lipidlowering therapies on cholinesterase activity in patients with coronary artery disease. Pharmacol Rep. 2017;69(1):150-5.

43. Stam-Slob MC, van der Graaf Y, Greving JP, et al. Cost-effectiveness of intensifying lipid-lowering therapy with statins based on individual absolute benefit in coronary artery disease patients. J Am Heart Assoc. 2017;6(2):e004648

44. Housholder-Hughes SD, Martin MM, McFarland MR, et al. Healthcare provider compliance with the 2013 ACC/AHA adult cholesterol guideline recommendation for high-intensity dose statins for patients with coronary artery disease. Heart Lung. 2017;46(4):328-333.

45. Lama S, Souraya D, Youssef F. Statin prescription strategies and atherogenic cholesterol goals attainment in Lebanese coronary artery disease patients. Int J Clin Pharm. 2017;39(4):919-26.

46. Protty MB, Lacey A, Hayes J, et al. Statins for secondary prevention: Clinical use in patients with acute coronary syndrome in Wales. Future Cardiol. 2017;13(2):137-41.

47. Turner RM, Yin P, Hanson A, et al. Investigating the prevalence, predictors, and prognosis of suboptimal statin use early after a non-ST elevation acute coronary syndrome. J Clin Lipidol. 2017;11(1):204-14.

48. Xie G, Sun Y, Myint PK, et al. Six-month adherence to Statin use and subsequent risk of major adverse cardiovascular events (MACE) in patients discharged with acute coronary syndromes. Lipids Health Dis. 2017;16(1): 155 .

49. Eisen A, Cannon CP, Braunwald E, et al. Predictors of nonuse of a high-potency statin after an acute coronary syndrome: Insights from the stabilization of plaques using darapladib-thrombolysis in myocardial infarction 52 (SOLID-TIMI 52) Trial. J Am Heart Assoc. 2017;6(1): e004332.

50. Serban MC, Colantonio LD, Manthripragada AD, et al. Statin intolerance and risk of coronary heart events and all-cause mortality following myocardial infarction. J Am Coll Cardiol. 2017;69(11):138695.

51. Lin H, Zhang YM. The effect of ezetimibe and simvastatin combination therapy on percutaneous coronary intervention patients. Int J Cardiol. 2017;242:1-3.

52. Takase S, Matoba T, Nakashiro S, et al. Ezetimibe in combination with statins ameliorates endothelial dysfunction in coronary arteries after stenting: The CuVIC Trial (Effect of Cholesterol Absorption Inhibitor Usage on Target Vessel Dysfunction After Coronary Stenting), a multicenter randomized controlled trial. Arterioscler Thromb Vasc Biol. 2017;37(2):350-8.

53. Hibi K, Sonoda S, Kawasaki M, et al. Effects of ezetimibe-statin combination therapy on coronary atherosclerosis in acute coronary syndrome. Circ J. 2018;82(3):757-66.

54. Lin Wu FL, Wang J, Ho W, et al. Effectiveness of a combination of ezetimibe and statins in patients with acute coronary syndrome and multiple comorbidities: A 6-year population-based cohort study. Int J Cardiol. 2017;233:43-51

55. Bohula EA, Wiviott SD, Giugliano RP, et al. Prevention of stroke with the addition of ezetimibe to statin therapy in patients with acute coronary syndrome in IMPROVE-IT. Circulation. 2017; 136(25):2440-50.

56. Ran D, Nie HJ, Gao YL, et al. A randomized, controlled comparison of different intensive lipid-lowering therapies in Chinese patients with non-STelevation acute coronary syndrome (NSTE-ACS): Ezetimibe and rosuvastatin versus high-dose rosuvastatin. Int J Cardiol. 2017;235:49-55.

57. Dai YY, Zhang HS, Zhang XG, et al. Statin-ezetimibe versus statin lipidlowering therapy in patients with acute coronary syndromes undergoing percutaneous coronary intervention. J Thorac Dis. 2017;9(5):1345-52.

58. Hagiwara N, Kawada-Watanabe E, Koyanagi R, et al. Low-density lipoprotein cholesterol targeting with pitavastatin+ezetimibe for patients with acute coronary syndrome and dyslipidaemia: The HIJPROPER study, a prospective, open-label, randomized trial. Eur Heart J. 2017;38(29):2264-76

59. Japaridze L, Sadunishvili M. The short-term effect of atorvastatin plus ezetimibe therapy versus atorvastatin monotherapy on clinical outcome in acute coronary syndrome patients by gender. Kardiol Pol. 2017;75(8):770-8.

60. Liu Z, Hao H, Yin C, et al. Therapeutic effects of atorvastatin and ezetimibe compared with double-dose atorvastatin in very elderly patients with acute coronary syndrome. Oncotarget. 2017;8(25):41582-9.

61. Wang J, Ai XB, Wang F, et al. Efficacy of ezetimibe combined with atorvastatin in the treatment of carotid artery plaque in patients with type 2 diabetes mellitus complicated with coronary heart disease. Int Angiol. 2017;36(5):467-73

62. Nelson JR, Wani O, May HT, et al. Potential benefits of eicosapentaenoic acid on atherosclerotic plaques. Vascul Pharmacol. 2017;91:1-9.

63. Nosaka K, Miyoshi T, Iwamoto M, et al. Early initiation of eicosapentaenoic acid and statin treatment is associated with better clinical outcomes than statin alone in patients with acute coronary syndromes: 1-year outcomes of a randomized controlled study. Int J Cardiol. 2017;228:173-9.

64. Watanabe T, Ando K, Daidoji H, et al. A randomized controlled trial of eicosapentaenoic acid in patients with coronary heart disease on statins. J Cardiol. 2017;70(6):537-44

65. Tani S, Nagao K, Yagi T, et al. Impact of adding Eicosapentaenoic acid to statin therapy on plasma Pentraxin 3 level in patients with stable coronary artery disease: A 6-month, randomized controlled study. Am J Cardiovasc Drugs. 2017;17(1):49-59.

66. Tani S, Nagao K, Kawauchi K, et al. The ratio of Eicosapentaenoic acid (EPA) to Arachidonic acid may be a residual risk marker in stable coronary artery disease patients receiving treatment with statin following EPA therapy. Am J Cardiovasc Drugs. 2017;17(5):409-20.

67. Marazzi G, Pelliccia F, Campolongo G, et al. Usefulness of nutraceutical (Armolipid Plus) versus ezetimibe and combination in statin-intolerant patients with dyslipidemia with coronary heart disease. Am J Cardiol. 2015;116(12):1798-801

68. Marazzi G, Campolongo G, Pelliccia F, et al. Comparison of low-dose statin versus low-dose statin+Armolipid Plus in high-intensity statinintolerant patients with a previous coronary event and percutaneous coronary intervention (ADHERENCE Trial). Am J Cardiol. 2017;120(6):893-7.

69. Cerit L, Duygu H, Gulsen K, et al. Effect of statins on coronary blood flow after percutaneous coronary intervention in patients with stable coronary artery disease. Neth Heart J. 2017;25(4):258-63.

70. Guo R, Yang L, Mu L, et al. Long-term statin use before primary percutaneous coronary intervention improves treatment outcomes of acute myocardial infarction. Exp Ther Med. 2017;13(4):1578-83.

71. Chen Y, Li D, Jing J, et al. Treatment trends, effectiveness, and safety of statins on lipid goal attainment in chinese percutaneous coronary intervention patients: a multicenter, retrospective cohort study. Clin Ther. 2017;39(9):1827-39.e1.

72. Lu C, Jia H, Wang Z. High-dose atorvastatin reduces the risk of cardiovascular events in patients with percutaneous coronary intervention. Oncotarget. 2017;8(41):70356-65. 
73. Ye Z, Lu H, Su Q, et al. Effect of high-dose rosuvastatin loading before percutaneous coronary intervention in Chinese patients with acute coronary syndrome: A systematic review and meta-analysis. PLoS One. 2017;12(2):e0171682.

74. Guo J, Zhang WZ, Zhao Q, et al. Study on the effect of different doses of rosuvastatin on ventricular remodeling in patients with acute coronary syndrome after emergency percutaneous coronary intervention. Eur Rev Med Pharmacol Sci. 2017;21(19):4457-63.

75. Wada H, Ogita M, Miyauchi K, et al. Impact of gender difference on long-term outcomes of percutaneous coronary intervention for coronary artery disease in patients under statin treatment. Heart Vessels. 2017;32(1):16-21.

76. Sawano M, Kohsaka S, Abe T, et al. Patterns of statin non-prescription in patients with established coronary artery disease: A report from a contemporary multicenter Japanese PCI registry. PLoS One. 2017;12(8):e0182687.

77. Natsuaki M, Morimoto T, Furukawa Y, et al. Effect of statin therapy on cardiovascular outcomes after coronary revascularization in patients $\geq$ 80 years of age: observations from the CREDO-Kyoto Registry Cohort-2. Atherosclerosis. 2014;237(2):821-8.

78. Ma H, Liu Y, Xie H, et al. The renoprotective effects of simvastatin and atorvastatin in patients with acute coronary syndrome undergoing percutaneous coronary intervention: An observational study. Medicine (Baltimore). 2017;96(32):e7351.

79. Briguori C, Quintavalle C, D’Alessio F, et al. Impact of statin therapy intensity on endothelial progenitor cells after percutaneous coronary intervention in diabetic patients. The REMEDY-EPC late study. Int J Cardiol. 2017;244:112-18.

80. Wang YB, Fu XH, Gu XS, et al. Effects of intensive pitavastatin therapy on glucose control in patients with non-ST elevation acute coronary syndrome. Am J Cardiovasc Dis. 2017;7(4):89-96.

81. Curtis M, Deng Y, Lee VV, et al. Effect of dose and timing of preoperative statins on mortality after coronary artery bypass surgery. Ann Thorac Surg. 2017;104(3):782-9

82. Liakopoulos OJ, Kuhn EW, Slottosch I, et al. Statin therapy in patients undergoing coronary artery bypass grafting for acute coronary syndrome. Thorac Cardiovasc Surg. 2017.

83. An J, Shi F, Liu S, et al. Preoperative statins as modifiers of cardiac and inflammatory outcomes following coronary artery bypass graft surgery: A meta-analysis. Interact Cardiovasc Thorac Surg. 2017;25(6):958-65.

84. Wang Y, Zhu S, DU R, et al. Statin initiation and renal outcomes following isolated coronary artery bypass grafting: A meta-analysis. J Cardiovasc Surg (Torino). 2017.

85. Nenna A, Lusini M, Spadaccio C, et al. Preoperative atorvastatin reduces bleeding and blood products use in patients undergoing on pump coronary artery bypass grafting. J Cardiovasc Med (Hagerstown). 2017;18(12):976-82.

86. Minami Y, Wang Z, Aguirre AD, et al. Clinical predictors for lack of favorable vascular response to statin therapy in patients with coronary artery disease: A serial optical coherence tomography study. J Am Heart Assoc. 2017;6(11):e006241.

87. Mori K, Ishida T, Tsuda S, et al. Enhanced Impact of Cholesterol Absorption Marker on New Atherosclerotic Lesion Progression After Coronary Intervention During Statin Therapy. J Atheroscler Thromb. 2017;24(2):123-32

88. Suwa S, Ogita M, Miyauchi K, et al. Impact of Lipoprotein (a) on longterm outcomes in patients with coronary artery disease treated with statin after a first percutaneous coronary intervention. J Atheroscler Thromb. 2017;24(11):1125-31. 